Modern Physics Letters A,

(C) World Scientific Publishing Company

\title{
ODD STATISTICS IN ODD DIMENSIONS FOR ODD COUPLINGS*
}

\author{
L. SRIRAMKUMAR ${ }^{\dagger}$ \\ Theoretical Physics Institute, Department of Physics, University of Alberta \\ Edmonton, Alberta T6G 2J1, Canada. \\ Received (received date) \\ Revised (revised date)
}

\begin{abstract}
We consider the response of a uniformly accelerated monopole detector that is coupled non-linearly to the $n$th power of a quantum scalar field in $(D+1)$-dimensional flat spacetime. We show that, when $(D+1)$ is even, the response of the detector in the Minkowski vacuum is characterized by a Bose-Einstein factor for all $n$. Whereas, when $(D+1)$ is odd, we find that a Fermi-Dirac factor appears in the detector response when $n$ is odd, but a Bose-Einstein factor arises when $n$ is even. We emphasize the point that, since, along the accelerated trajectory, the Wightman function and, as a result, the $(2 n)$-point function satisfy the Kubo-Martin-Schwinger condition (as required for a scalar field) in all dimensions, the appearance of a Fermi-Dirac factor (instead of the expected Bose-Einstein distribution) for odd $(D+1)$ and $n$ reflects a peculiar feature of the detector rather than imply a fundamental change in field theory.
\end{abstract}

\section{Introduction}

It has been a quarter of a century now since it was discovered that the response of a uniformly accelerated monopole detector that is coupled to a quantized massless scalar field is characterized by Planckian distribution when the field is assumed to be in the Minkowski vacuuml 3 . However, about a decade after the original discovery, it was noticed that this result is true only in even-dimensional flat spacetimes and it was pointed out that a Fermi-Dirac factor (rather than a Bose-Einstein factor) appears in the response of the accelerated detector when the dimensionality of spacetime is odd (see Refs. The detector due to Unruh and DeWitt is coupled linearly to the quantum scalar field. During the last decade or so, motivated by different reasons, there has been an occasional interest in literature in studving the response of detectors that are coupled non-linearly to the quantum field 12 . 13,4 , 15 . 16 . It will be interesting to examine whether the non-linearity of the coupling affects the result in odd-dimensional flat spacetimes that we mentioned above.

*This title is motivated by the title of an unpublished paper by C. R. Stephenst.

${ }^{\dagger}$ Present address: Harish-Chandra Research Institute, Chhatnag Road, Jhunsi, Allahabad 211 019, India. E-mail: sriram@mri.ernet.in. 
In this note, we shall consider the response of a uniformly accelerated monopole detector that is coupled to the $n$th power (with $n$ being a positive integer) of a quantum scalar field in $(D+1)$-dimensional flat spacetime. As we shall see, the non-linearity of the detector's coupling affects the afore-mentioned result in odd spacetime dimensions in an interesting fashion. We shall show that, when $(D+1)$ is even, a Bose-Einstein factor arises in the response of the detector for all $n$, whereas, when $(D+1)$ is odd, a Fermi-Dirac factor appears in the detector response when $n$ is odd, but a Bose-Einstein factor arises when $n$ is even.

We shall adopt units such that $\hbar=c=1$ and we shall denote the set of $(D+1)$ coordinates $x^{\mu}$ as $\tilde{x}$.

\section{The Non-linearly Coupled Detector}

Consider a monopole detector that is moving along a trajectory $\tilde{x}(\tau)$, where $\tau$ is the proper time in the frame of the detector. Let the detector interact with a real scalar field $\Phi$ through the non-linear interaction Lagrangian 15

$$
\mathcal{L}_{\mathrm{int}}=\bar{c} m(\tau) \Phi^{n}[\tilde{x}(\tau)]
$$

where $\bar{c}$ is a small coupling constant, $m(\tau)$ is the detector's monopole moment and $n$ is a positive integer that denotes the index of non-linearity of the coupling. Let us now assume that the quantum field $\hat{\Phi}$ is initially in the vacuum state $|0\rangle$ and the detector is in its ground state $\left|E_{0}\right\rangle$ corresponding to an energy eigen value $E_{0}$. Then, up to the first order in perturbation theory, the amplitude of transition of the non-linearly coupled detector to an excited state $|E\rangle$, corresponding to an energy eigen value $E$ ( $>E_{0}$ ), is described by the integral (see, for e.g., Ref.17)

$$
\mathcal{A}_{n}(\mathcal{E})=\mathcal{M} \int_{-\infty}^{\infty} d \tau e^{i \mathcal{E} \tau}\left\langle\Psi\left|\hat{\Phi}^{n}[\tilde{x}(\tau)]\right| 0\right\rangle
$$

where $\mathcal{M} \equiv\left(i \bar{c}\left\langle E|\hat{m}(0)| E_{0}\right\rangle\right), \mathcal{E}=\left(E-E_{0}\right)>0$ and $|\Psi\rangle$ is the state of the quantum scalar field after its interaction with the detector. (Since the quantity $\mathcal{M}$ depends only on the internal structure of the detector and does not depend on its motion, we shall drop this quantity hereafter.)

The transition amplitude $\mathcal{A}_{n}(\mathcal{E})$ above involves products of the quantum field $\hat{\Phi}$ at the same spacetime point and, hence, we will encounter divergences when evaluating this transition amplitude. In order to avoid the divergences, we shall normal order the operators in the matrix element in the transition amplitude $\mathcal{A}_{n}(\mathcal{E})$ with respect to the Minkowski vacuum 15 . That is, we shall assume that the transition amplitude (2) above is instead given by the expression

$$
\overline{\mathcal{A}}_{n}(\mathcal{E})=\int_{-\infty}^{\infty} d \tau e^{i \mathcal{E} \tau}\left\langle\Psi\left|: \hat{\Phi}^{n}[\tilde{x}(\tau)]:\right| 0\right\rangle
$$


where the colons denote normal ordering with respect to the Minkowski vacuum. Then, the transition probability of the detector to all possible final states $|\Psi\rangle$ of the quantum field is given by

$$
\mathcal{P}_{n}(\mathcal{E})=\sum_{|\Psi\rangle}\left|\overline{\mathcal{A}}_{n}(\mathcal{E})\right|^{2}=\int_{-\infty}^{\infty} d \tau \int_{-\infty}^{\infty} d \tau^{\prime} e^{-i \mathcal{E}\left(\tau-\tau^{\prime}\right)} G^{(n)}\left[\tilde{x}(\tau), \tilde{x}\left(\tau^{\prime}\right)\right]
$$

where $G^{(n)}\left[\tilde{x}(\tau), \tilde{x}\left(\tau^{\prime}\right)\right]$ is the $(2 n)$-point function defined as

$$
G^{(n)}\left[\tilde{x}(\tau), \tilde{x}\left(\tau^{\prime}\right)\right]=\left\langle 0\left|: \hat{\Phi}^{n}[\tilde{x}(\tau)]:: \hat{\Phi}^{n}\left[\tilde{x}\left(\tau^{\prime}\right)\right]:\right| 0\right\rangle .
$$

In cases wherein the $(2 n)$-point function $G^{(n)}\left(\tau, \tau^{\prime}\right)\left(\equiv G^{(n)}\left[\tilde{x}(\tau), \tilde{x}\left(\tau^{\prime}\right)\right]\right)$ is invariant under time translations in frame of the detector, we can define a transition probability rate for the detector as follows:

$$
\mathcal{R}_{n}(\mathcal{E})=\int_{-\infty}^{\infty} d \bar{\tau} e^{-i \mathcal{E} \bar{\tau}} G^{(n)}(\bar{\tau})
$$

where $\bar{\tau}=\left(\tau-\tau^{\prime}\right)$.

\section{Odd Statistics in Odd dimensions for Odd Couplings}

Let us now assume that the quantum scalar field $\hat{\Phi}$ is in the Minkowski vacuum. In such a case, the $(2 n)$-point function $G^{(n)}\left(\tilde{x}, \tilde{x}^{\prime}\right)$ simplifies to

$$
G_{\mathrm{M}}^{(n)}\left(\tilde{x}, \tilde{x}^{\prime}\right)=(n !)\left[G_{\mathrm{M}}^{+}\left(\tilde{x}, \tilde{x}^{\prime}\right)\right]^{n},
$$

where $G_{\mathrm{M}}^{+}\left(\tilde{x}, \tilde{x}^{\prime}\right)$ denotes the Wightman function in the Minkowski vacuum. In $(D+1)$ spacetime dimensions (and for $(D+1) \geq 3$ ), the Wightman function for a massless scalar field in the Minkowski vacuum is given by (see, for instance, Refs.

$$
G_{\mathrm{M}}^{+}\left(\tilde{x}, \tilde{x}^{\prime}\right)=\mathcal{C}_{D}\left[(-1)\left(\left(t-t^{\prime}-i \epsilon\right)^{2}-\left|\mathbf{x}-\mathbf{x}^{\prime}\right|^{2}\right)\right]^{-(D-1) / 2},
$$

where $\epsilon \rightarrow 0^{+},\left[t, \mathbf{x} \equiv\left(x^{1}, x^{2}, \ldots, x^{D}\right)\right]$ are the Minkowski coordinates and the quantity $\mathcal{C}_{D}$ is given by

$$
\mathcal{C}_{D}=\left(4 \pi^{(D+1) / 2}\right)^{-1} \Gamma[(D-1) / 2]
$$

with $\Gamma[(D-1) / 2]$ denoting the Gamma function.

${ }^{a}$ It ought to be noted here that we would have arrived at the expression ( (7) for the (2n)-point function in the Minkowski vacuum even if we had started with the transition amplitude (2) (instead of the normal ordered amplitude (3)), rewritten the resulting (2n)-point function in the transition probability in terms of the two-point functions using Wick's theorem and then replaced the divergent terms that arise (i.e. those two-point functions with coincident points) with the corresponding regularized expressions (for a discussion on this point, also see Ref.15). 
Now, consider a detector accelerating uniformly along the $x^{1}$ direction with a proper acceleration $g$. The trajectory of such a detector is given by (see, for e.g., Ref 17)

$$
t(\tau)=g^{-1} \sinh (g \tau), x^{1}(\tau)=g^{-1} \cosh (g \tau), x^{2}=x^{3}=\ldots=x^{D}=0,
$$

where $\tau$ is the proper time in the frame of the detector. On substituting this trajectory in the Minkowski Wightman function (8), we obtain that (see, for instance, Refs.5, 8.9$)$

$$
G_{\mathrm{M}}^{+}(\bar{\tau})=\left[\mathcal{C}_{D}(g / 2 i)^{(D-1)}\right](\sinh [(g \bar{\tau} / 2)-i \epsilon])^{-(D-1)}
$$

Therefore, along the trajectory of the uniformly accelerated detector, the $(2 n)$-point function in the Minkowski vacuum (7) is given by

$$
G_{\mathrm{M}}^{(n)}(\bar{\tau})=(n !)\left[\mathcal{C}_{D}^{n}(g / 2 i)^{\alpha}\right](\sinh [(g \bar{\tau} / 2)-i \epsilon])^{-\alpha}
$$

where $\alpha=[(D-1) n]$.

On substituting the $(2 n)$-point function (12) in the expression (6) and carrying out the resulting integral, we find that the transition probability rate of the uniformly accelerated, non-linearly coupled detector can be written as (cf. Ref.ts, p. 305, Eq. 3.314; p. 950, Eq. 8.384.1; p. 937, Eqs. 8.331, 8.332.1 and 8.332.2)

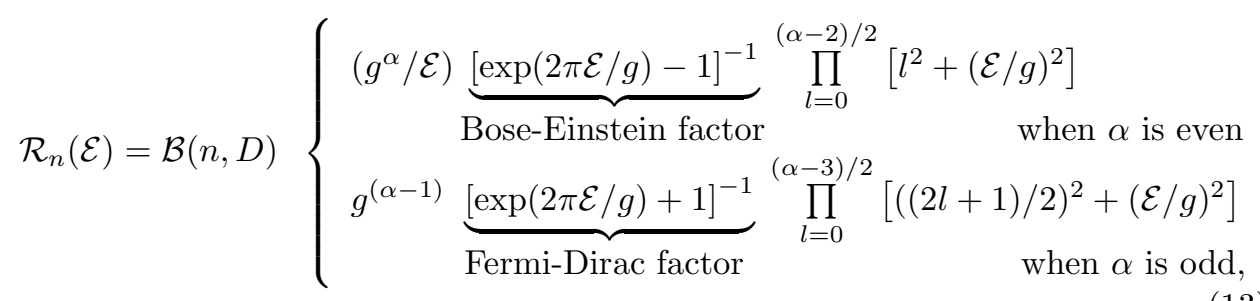

where the quantity $\mathcal{B}(n, D)$ is given by

$$
\mathcal{B}(n, D)=(2 \pi)(n !)\left[\mathcal{C}_{D}^{n} / \Gamma(\alpha)\right]
$$

When $(D+1)$ is even, $\alpha$ is even for all $n$ and, hence, a Bose-Einstein factor will always arise in the response of the uniformly accelerated detector in an evendimensional flat spacetime. Whereas, when $(D+1)$ is odd, evidently, $\alpha$ will be odd or even depending on whether $n$ is odd or even. Therefore, in an odd-dimensional flat spacetime, a Fermi-Dirac factor will arise in the detector response when $n$ is odd (as in the case of the Unruh-DeWitt detector), but a Bose-Einstein factor will appear when $n$ is even!

A few remarks regarding this curious result are in order. Firstly, the temperature associated with the Bose-Einstein and the Fermi-Dirac factors that appear in the response of the non-linearly coupled detector is the standard Unruh temperature, 
viz. $(g / 2 \pi)$. Secondly, the response of the detector is characterized completely by either a Bose-Einstein or a Fermi-Dirac distribution only in situations wherein $\alpha<$ 3 . For cases such that $\alpha \geq 3$, the detector response contains, in addition to a BoseEinstein or a Fermi-Dirac factor a term which is polynomial in $(\mathcal{E} / g)$. Thirdly, in Figs. 1 and 2, following Unruhl, we have plotted the transition probability rate of the detector (in fact, the quantity $\overline{\mathcal{R}}_{n}(\mathcal{E})=\left[\mathcal{R}_{n}(\mathcal{E}) / \mathcal{R}_{n}(0)\right]$ ) for a few different values of $D$ for the case $n=3$ and for a few different values of $n$ for the case wherein $(D+1)=3$, respectively. (In plotting these figures, we have set $g=(2 \pi)$.) It is

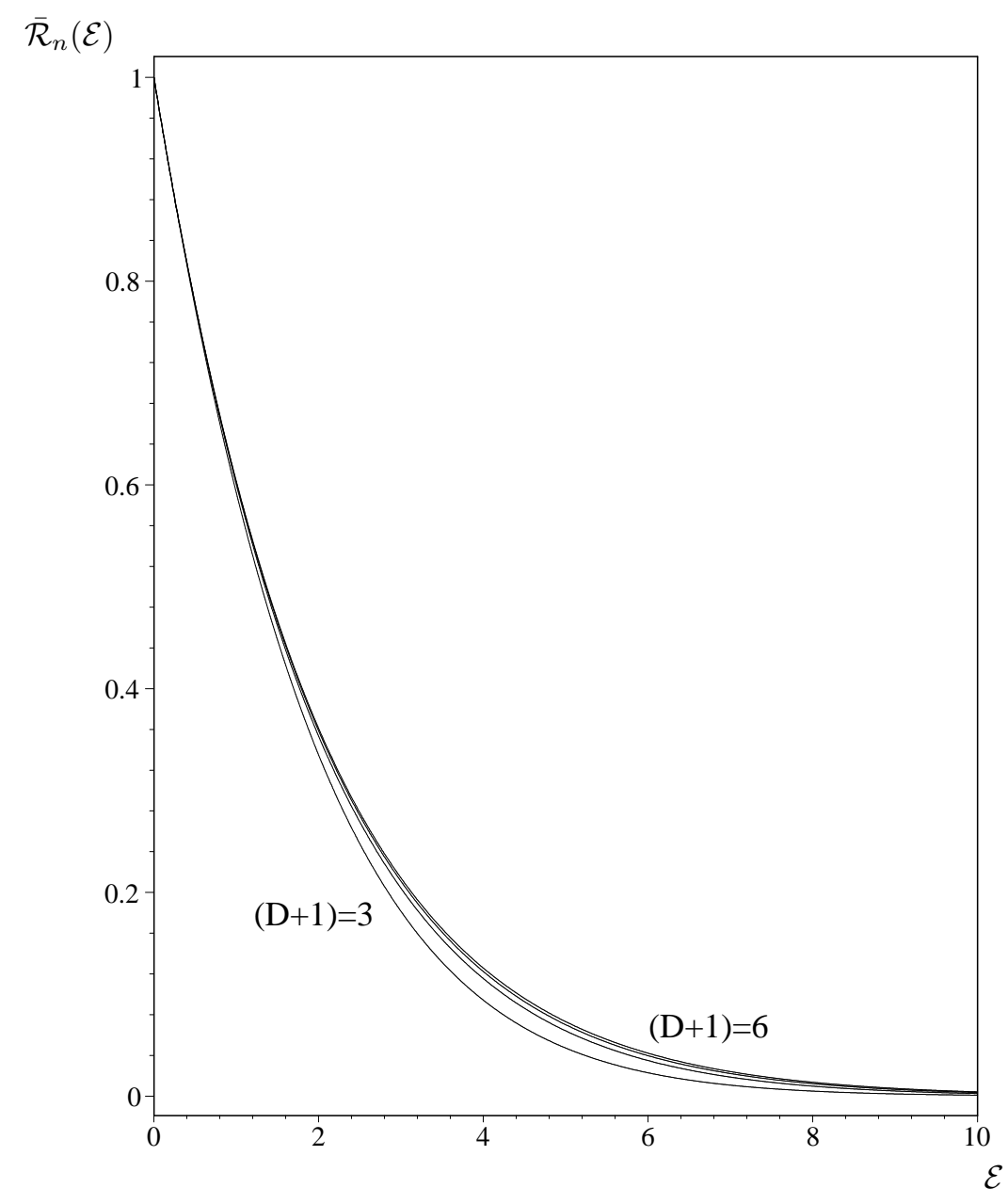

Fig. 1. $\overline{\mathcal{R}}_{n}(\mathcal{E})$ vs. $\mathcal{E}$ for $n=3$ and $(D+1)=3,4,5,6$.

interesting to note from these two figures that, though the characteristic response of the detector alternates between the Bose-Einstein and the Fermi-Dirac factors as 


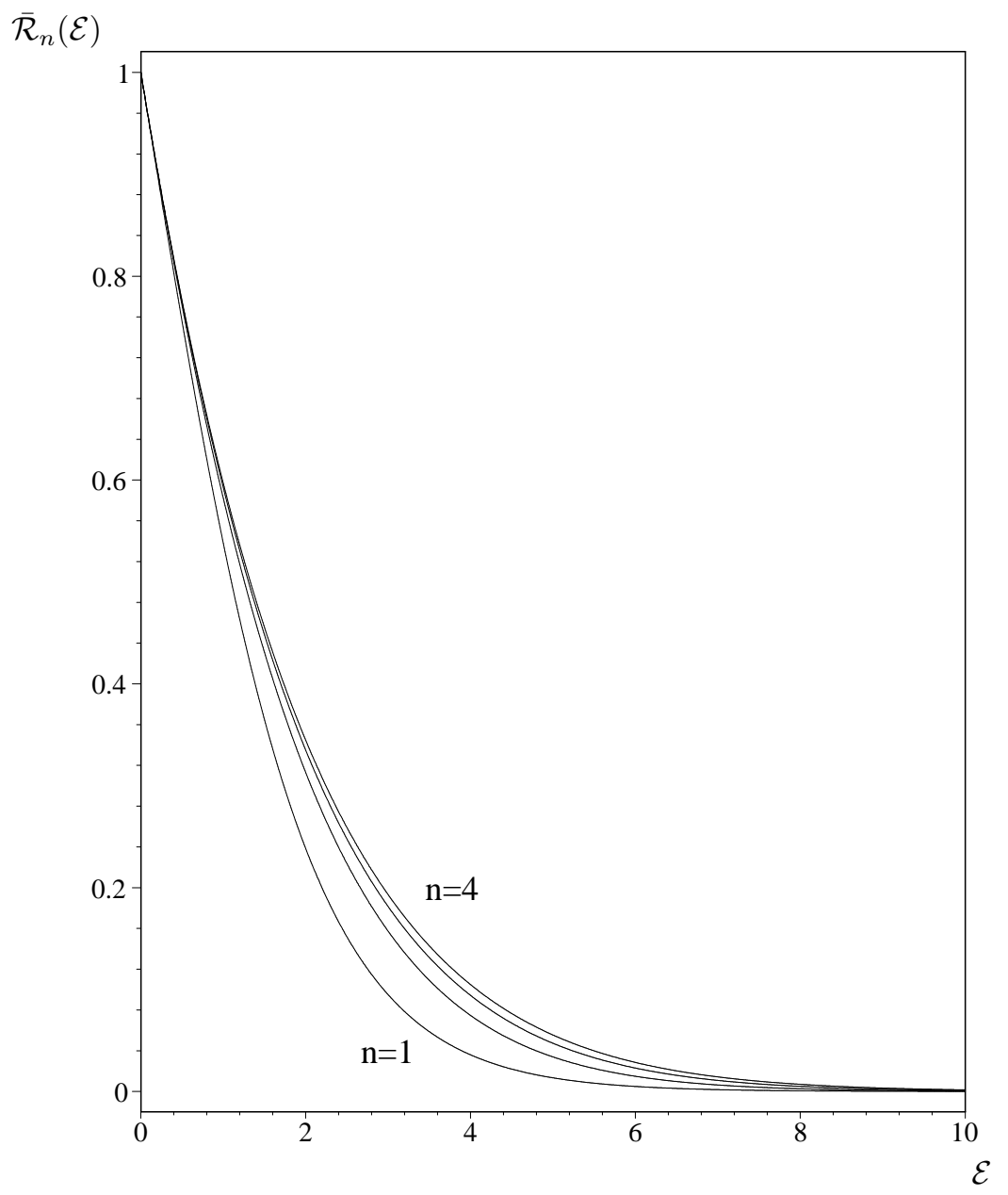

Fig. 2. $\overline{\mathcal{R}}_{n}(\mathcal{E})$ vs. $\mathcal{E}$ for $(D+1)=3$ and $n=1,2,3,4$. 
we go from one $D$ to another for odd $n$ (or from one $n$ to another when $(D+1)$ is odd), the complete spectra themselves exhibit a smooth dependence both on the index of non-linearity of the coupling and the dimension of spacetime.

\section{Apparent nature of the odd statistics}

The point that needs to be emphasized regarding the result we have obtained above is the apparent nature of the "inversion of statistics" that arises in odd dimensions for odd couplings. It is easy to see that, in the frame of the uniformly accelerated detector, the Wightman function in the Minkowski vacuum (11) is skew-periodic in imaginary proper time with a period corresponding to the inverse of the Unruh temperature, i.e.

$$
G_{\mathrm{M}}^{+}(\bar{\tau})=G_{\mathrm{M}}^{+}[-\bar{\tau}+i(2 \pi / g)] .
$$

Also, this property holds for all $D$. In other words, along the accelerated trajectory, the Wightman function satisfies the Kubo-Martin-Schwinger (KMS) condition as required for a Bosonic field in all dimensions (for a discussion on the KMS condition, see, for e.g., Ref 9 , Chaps. 4 and 5). Since the (2n)-point function in the Minkowski vacuum is proportional to the $n$th power of the Wightman function, it is then obvious that, in the frame of the accelerated detector, the $(2 n)$-point function will also be skew-periodic in imaginary proper time, thereby satisfying the KMS condition (as required for a Bosonic field) for all $D$ and $n$ (cf. Eq. (12)). Had the appearance of the Fermi-Dirac factor for odd $(D+1)$ and $n$ been the manifestation of a truly fundamental change in the field theory, then, in such situations, the $(2 n)$-point function along the accelerated trajectory would have been skew as well as anti-periodic in imaginary proper time as expected of a Fermionic field (see, for instance, Ref.7). The fact that this does not occur then clearly implies that the appearance of the Fermi-Dirac factor (instead of the expected Bose-Einstein factor) for odd $(D+1)$ and $n$ just reflects a peculiar feature of the detector rather than suggest a fundamental shift in the field theory.

\section{Outlook}

The appearance of a Fermi-Dirac factor (instead of the expected Planckian distribution) is known to occur in the response of a comoving Unruh-DeWitt detector (that is coupled to a massless scalar field) in odd-dimensional de Sitter spacetimes

(see, for e.g., Refs. 1.29; for a recent discussion, see Ref29) and also in the case of a detector stationed at a constant radius in the spacetime of the $(2+1)$ dimensional Banados-Teitelboim-Zanelli (BTZ) black hole21. Moreover, it has recently been pointed out that, not only comoving, but even accelerated Unruh-DeWitt detectors in de Sitter spacetime as well as accelerated detectors (with proper acceleration bevond a certain critical value) in anti-de Sitter spacetime exhibit a thermal response22 23.24.25. It will be interesting to investigate as to how the non-linearity of the detector's coupling would affect the response of a static detector around the BTZ black hole and also the response of comoving as well as accelerated detectors 
in de Sitter and anti-de Sitter spacetimes in different dimensions. Furthermore, it has been shown that a similar "inversion of statistics" occurs in the response of a monopole detector that is coupled linearly to the scalar density of a massless Dirac field in odd-dimensional flat and de Sitter spacetimed9.11.19.20 and also around the BTZ black hole26, i.e. the response of the detector exhibits a Bose-Einstein factor instead of the Fermi-Dirac factor expected in such situations. It will be worthwhile to examine as to how detectors coupled non-linearly to the scalar density of spinor fields respond in odd-dimensional spacetimes. We plan to address these issues in some detail in a forthcoming publicatione7.

\section{Acknowledgments}

We would like to thank Don Page, Valeri Frolov and Jonathan Oppenheim for discussions, William Unruh for correspondence and discussions, Andrei Zelnikov for comments on an earlier version of the manuscript and Supratim Sengupta for help with Maple. We would also like to thank Christopher Stephens for correspondence and for providing a copy of his unpublished paper. We also wish to thank the organizers of IGQR-I for their invitation to speak at the Workshop. This work was supported by the Natural Sciences and Engineering Research Council of Canada.

\section{References}

1. C. R. Stephens, Odd statistics in odd dimensions, University of Maryland Report, 1985 (unpublished).

2. W. G. Unruh, Phys. Rev. D 14, 870 (1976).

3. B. S. DeWitt, Quantum gravity: The new synthesis, in General Relativity: An Einstein Centenary Survey, eds. S. W. Hawking and W. Israel (Cambridge University Press, Cambridge, England, 1979).

4. S. Takagi, Prog. Theor. Phys. 72, 505 (1984).

5. S. Takagi, Prog. Theor. Phys. 74, 142 (1985).

6. C. R. Stephens, On Some Aspects of the Relationship Between Quantum Physics, Gravity and Thermodynamics, PhD thesis, University of Maryland, 1986.

7. H. Ooguri, Phys. Rev. D 33, 3573 (1986).

8. W. G. Unruh, Phys. Rev. D 34, 1222 (1986).

9. S. Takagi, Prog. Theor. Phys. Suppl. 88, 1 (1986).

10. J. R. Anglin, Phys. Rev. D 47, 4525 (1993).

11. H. Terashima, Phys. Rev. D 60, 084001 (1999).

12. K. J. Hinton, J. Phys. A: Math. Gen. 16, 1937 (1983).

13. K. J. Hinton, Class. Quantum Grav. 1, 27 (1984).

14. T. Padmanabhan and T. P. Singh, Class. Quantum Grav. 4, 1397 (1987).

15. N. Suzuki, Class. Quantum Grav. 14, 3149 (1997).

16. L. Sriramkumar, Mod. Phys. Lett. A 14, 1869 (1999).

17. N. D. Birrell and P. C. W. Davies, Quantum Fields in Curved Space (Cambridge University Press, Cambridge, England, 1982), Sec. 3.3.

18. I. S. Gradshteyn and I. M. Ryzhik, Table of Integrals, Series and Products (Academic Press, New York, 1980).

19. S. Takagi, Prog. Theor. Phys. 74, 501 (1985).

20. T. Murata, K. Tsunoda and K. Yamamoto, gr-qc/0102080. 
21. G. Lifschytz and M. Ortiz, Phys. Rev. D 49, 1929 (1994).

22. S. Deser and O. Levin, Class. Quantum Grav. 14, L163 (1997).

23. T. Jacobson, Class. Quantum Grav. 15, 251 (1998).

24. S. Deser and O. Levin, Class. Quantum Grav. 15, L85 (1998).

25. S. Deser and O. Levin, Phys. Rev. D 59, 064004 (1999).

26. S. Hyun, Y.-S. Song and J. H. Yee, Phys. Rev. D 51, 1787 (1995).

27. L. Sriramkumar, Detecting thermal effects in spacetimes with horizons, In preparation. 